\title{
DERIVATION OF A NEW EXPRESSION RELATING THE ION DENSITY TO THE ION CURRENT FROM A LANGMUIR PROBE
}

\author{
by J. VAN ECK and H. M. J. KINDERDIJK \\ Fysisch Laboratorium der Rijksuniversiteit, Utrecht, Nederland
}

\section{Synopsis}

For a plasma, consisting of electrons and singly ionized ions an expression is derived relating the ion current, at a given probe voltage, to the ion density. The derivation is valid in the limiting case of low ion temperarure $\left(T_{i} \ll T_{e}\right)$, a high value of the negative probe-potential $\left(\left|q V_{p}\right| \gg k T_{e}\right)$ and a long mean free path. The validity of the formula is discussed. The results are compared with computer calculations of different authors and the difference is within $10 \%$ in the considered range. The ion densities, calculated with our formula, agree very well with electron densities, calculated from the same probe characteristics (standard deviation 10\%), taking into account the secondary emission of the ions. The measurements are performed in the positive column of a helium discharge.

I. Introduction. Until recently it was difficult to calculate the ion density from measurements of the ion current. Although the Poisson equation governing this problem has been solved by several people $\left.\left.{ }^{1}\right)^{2}\right)^{3}$ ) with the help of electronic computers, they give their results in graphs which have the difficulty that one must interpolate from small figures. We have derived a formula which gives a direct analytical relation between the ion current and the ion density at a given probe voltage, if the electron temperature is known.

To find this relation, we divide the surroundings of the probe into three different regions (see fig. 1):

I. The positive space-charge layer, where mostly ions are present.

II. The reflection layer. This layer contains ions and a minority of electrons which are reflected.

III. The pseudo plasma, where equal amounts of ions and electrons are present; the density is, however, somewhat lower than in the undisturbed plasma.

At the boundary, between region II and III, we know the relation between ion concentration and current density. To connect the probe current with the current density at this boundary, we must know the outer radius of the 
reflection layer (II). This radius is connected to the radius of the positive space-charge layer (I) following a method given by Kagan and Perel ${ }^{4}$ ). An approximation of the radius function $\left\{-\alpha\left(x_{0} / x_{p}\right)\right\}^{2}$ in the "three-halves" space-charge law of Child-Langmuir ${ }^{5}$ ), for the ion layer around the probe, gives us the possibility to construct a formula which gives an explicit relation between the probe current, probe voltage, density of ions, and electron temperature.

The results are compared with computer calculations of Allen ${ }^{1}$ ), Bernstein and Rabinowit $z^{2}$ ), and Chen ${ }^{3}$ ). Measurements of ion and electron densities are performed in the positive column of a helium discharge and compared to each other.

II. 1. Derivation of the dimensionless Poisson equation. Let us consider a spherical probe with radius $r_{p}$ immersed in a plasma of electrons and single ionized positive ions. We start from the following conditions:

$T_{e} \gg T_{i}, q V \gg k T_{e}$ and $\lambda \gg$ diameter of the range, where the plasma is disturbed in which.

$T_{e}=$ electron tempcraturc

$T_{i}=$ ion temperature

$V=$ negative potential on the probc

$q=$ electronic charge

$k=$ constant of Boltzmann

$\lambda=$ mean free path of ions.

These are the usual conditions existing in discharge tubes at low pressure. If the electron distribution is taken to be Maxwellian, we know that the electron density $n_{e}$ follows the Boltzmann law:

$$
n_{e}=n_{0} \exp \left(-q V / k T_{e}\right)
$$

in which $n_{0}=$ the density of the undisturbed plasma.

When the ions have reached energies which are much larger than the initial energies because of the potential drop around the probe, their velocity will be nearly: $\left(-2 q V / m_{i}\right)^{0.5}$. As a consequence the ion density $n_{i}$ can be expressed in the form

$$
n_{i}=i^{+} / q .4 \pi r^{2}\left(2 q V m_{i}^{-1}\right)^{0.5}
$$

if $V \gg V_{0}$ (the kinetic energy of the ions).

In this expression $m_{i}=$ ion mass; $i^{+}=$ion current to the probe; $r=$ radial coördinate.

The potential distribution, in the vicinity of the probe, is described by Poisson's equation:

$$
\varepsilon_{0} \nabla_{r}^{2} V=q\left(n_{i}-n_{e}\right) .
$$

We substitute $n_{e}$ and $n_{i}$ from (1) and (2) into (3) and define the following 
dimensionless quantities:

$x=r / \Lambda$ in which $\Lambda=\left(\varepsilon_{0} k T_{e} / q^{2} n_{0}\right)^{0.5}$ : Debye length for electrons in a plasma. $y=q V \mid k T_{e}$

$p=i^{+} / I_{A}$ in which $I_{A}=\varepsilon_{0}\left(k T_{e} / q\right)^{0.5}\left(2 q / m_{i}\right)^{1.5}$ : Drift current through a Debye area $A^{2}$ from ions accelerated to the electron temperature.

Then we get a dimensionless relation between $x$ and $y$ with $p$ as a parameter:

$$
\nabla_{x}^{2} y=p / 4 \pi x^{2} y^{0.5}-\exp (-y)
$$

Equation (4) has been solved by Allen ${ }^{1}$ ) and more recently by $\mathrm{Chen}^{3}$ ) with help of a computer. They give their results only in graphs.

II. 2. Derivation of an explicit relation for the radius of the ion sheath. We consider the case that only ions are present. The Poisson equation (4) then reduces to

$$
\nabla^{2} y=p / 4 \pi x^{2} y^{0.5}
$$

because the electron concentration $n_{e}$ is taken zero (see equation (3)). Langmuir e.a. ${ }^{5}$ ) have solved this problem for two concentric spheres using the following boundary conditions:

$1^{\circ}$. $V$ and $\mathrm{d} V / \mathrm{d} r=0$ for $r=r_{0}$ at the outer sphere

$2^{\circ}$. zero initial velocity

$3^{\circ}$. $V=V_{p}$ for $r=r_{p}$ at the inner sphere ( $p$ refers to the probe; 0 refers to the outer sphere).

In our case, in a plasma, the first two conditions are not fulfilled; however, $\mathrm{d} V / \mathrm{d} r$ and the initial velocity are small because the greater part of the potential fall is within this ion layer. So we use the given solution which reads in dimensionless parameters:

$$
\left(y_{p}-y_{0}\right)^{1.5}=9 p / 16 \pi\left\{-\alpha\left(x_{0} / x_{p}\right)\right\}^{2}
$$

in which $y_{0}$ is taken zero.

The function $\left\{-\alpha\left(x_{0} / x_{p}\right)\right\}^{2}$ is calculated by Langmuir $\left.{ }^{5}\right)$ and Ollendorf $\left.{ }^{6}\right)$. We want an explicit relation between $x_{0} / x_{p}, y$ and $p$. As is shown in fig. 2, we approximate the curve $\log (-\alpha)^{2}$ versus $\log \left(x_{0} / x_{p}-1\right)$ by three straight lines, corresponding respectively to solutions of the space charge equation with plane- and more or less pronounced spherical geometry. A good approximation, within $3 \%$, is possible in the range $1.6<x_{0} / x_{p}<6$ by:

$$
(-\alpha)^{2}=0.75\left(x_{0} / x_{p}-1\right)^{1.72}
$$

and within $5 \%$ in the range $6<x_{0} / x_{p}<200$ by:

$$
(-\alpha)^{2}=\left(x_{0} / x_{p}-1\right)^{1.55} \text {. }
$$

In the following we use equation $7 a$, because the sheath thickness is within this region. Using equation $7 b$, the results are not much different. 
II. 3. Plasma equation and boundary of the ion layer. The plasma equation is obtained by considering the plasma as quasi neutral ${ }^{5}$ ). In this case the ion and clcctron density are almost equal. This reduces formula (4) to

$$
p \approx 4 \pi x^{2} y^{0.5} \exp (-y)
$$

By differentiating equation (8) one finds that $\mathrm{d} y / \mathrm{d} x \rightarrow \infty$ for $y \rightarrow 0.5$ (see fig. 1). It is clear that for $y \rightarrow 0.5$ the plasma equation will deviate from the

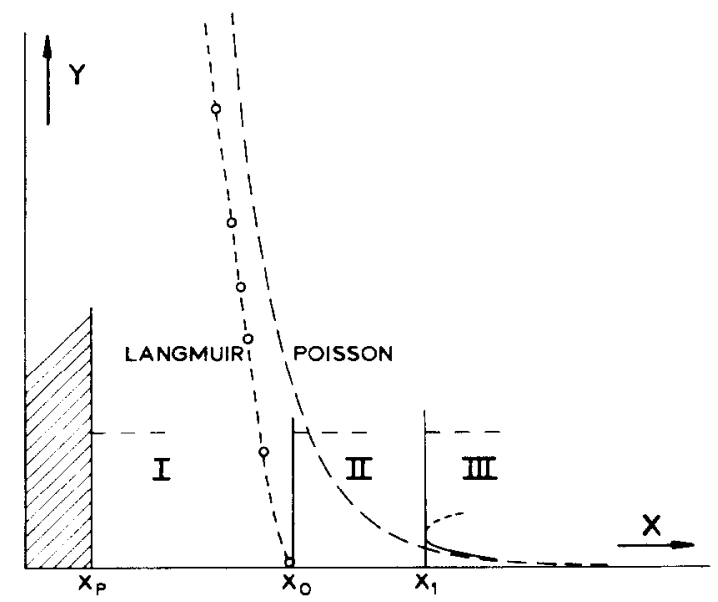

Fig. 1. Comparison of the Child-Langmuir-, Poisson- and plasma equation.

A division is made in three regions:

I. Space charge layer (Langmuir sheath)

$\left.\begin{array}{l}\text { II. Reflection layer } \\ \text { III. Pseudo plasma }\end{array}\right\}$ (pre-sheath)

solution of the Poisson equation, but as is shown in table $\mathrm{I}$, the value of $\exp (-y) \cdot y^{0.5}$ (which is associated with the current density) is quite insensitive to the value of $y$.

Because we are interested in the value of $x$ at the boundary of the plasma,

TABLE I

\begin{tabular}{|c|c|c|c|c|c|c|c|c|c|c|c|c|}
\hline$y$ & 0 & 0.10 & 0.15 & 0.20 & 0.25 & 0.30 & 0.40 & 0.50 & 0.60 & 0.70 & 0.80 & 1.0 \\
\hline $\exp (-y) \cdot y^{0.5}$ & 0 & 0.28 & 0.34 & 0.37 & 0.39 & 0.41 & 0.42 & 0.43 & 0.42 & 0.41 & 0.40 & 0.37 \\
\hline
\end{tabular}

we break off the plasma solution at $y=0.3$ and find, within $10 \%$, the same value for $x_{1}^{2}$ as is found for $y=0.5$. Taking $y_{1}$ to be 0.5 we find for $x_{1}^{2}$

$$
x_{1}^{2}=p / 4 \pi \exp \left(-y_{1}\right) y_{1}^{0.5}=0.43^{-1} p / 4 \pi \text {. }
$$

Inside the sheath, the potential increases rapidly on approaching the probe and the density of electrons and ions decreases. However, the electron density decreases much more rapidly then the ion density. As a consequence 
we are not able to define a sharp boundary from the $3 / 2$ power law (equation 6) but we have to find another criterion.

Kagan and Perel suppose that the charge density has a maximum at a certain pair of values $y_{2}$ and $x_{2}$ of the potential and radius (see fig. 3). This maximum is caused by the fact that the electrons are repelled and the ions attracted. They take this maximum to be the boundary of the space charge layer of the ions.

We have calculated the space charge with the help of computer curves

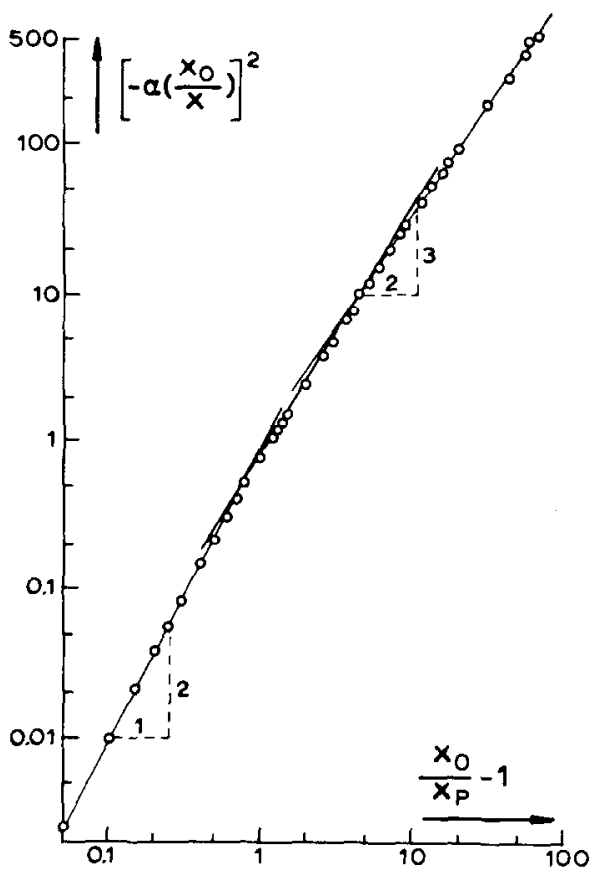

Fig. 2. The curve is divided in 3 parts: For $x_{0} / x_{p}<2$ the plane geometry solution of the space charge layer is found. For $x_{0} / x_{p}>6$ the spherical vacuum condensator solution is a good approximation. In the transition region we find

$$
(-d)^{2}=0.75\left(x_{0} / x_{p}-1\right)^{1.72} \text {. }
$$

published by Allen, and Bernstein and Rabinowitz, for several values of $p / 4 \pi$. As is shown in fig. 3 we have found that the maximum, or only a bend in the curves, depends on the current, so that the criterion of Kagan is not valid for all cases. However, we use this criterion to calculate the boundary of the ion layer, because a better one is not available. If there is a maximum or a bend in a plot of the charge density we find there:

$$
\mathrm{d} \rho / \mathrm{d} x=0 \text { or } \quad \partial \rho / \partial x+\partial \rho / \partial x \cdot \mathrm{d} y / \mathrm{d} x=0 .
$$

With the equation (1) and (2) and the criterion (10) we calculate a function $y=f(x)$, which gives, for a certain value of $p$, a set of corresponding values 


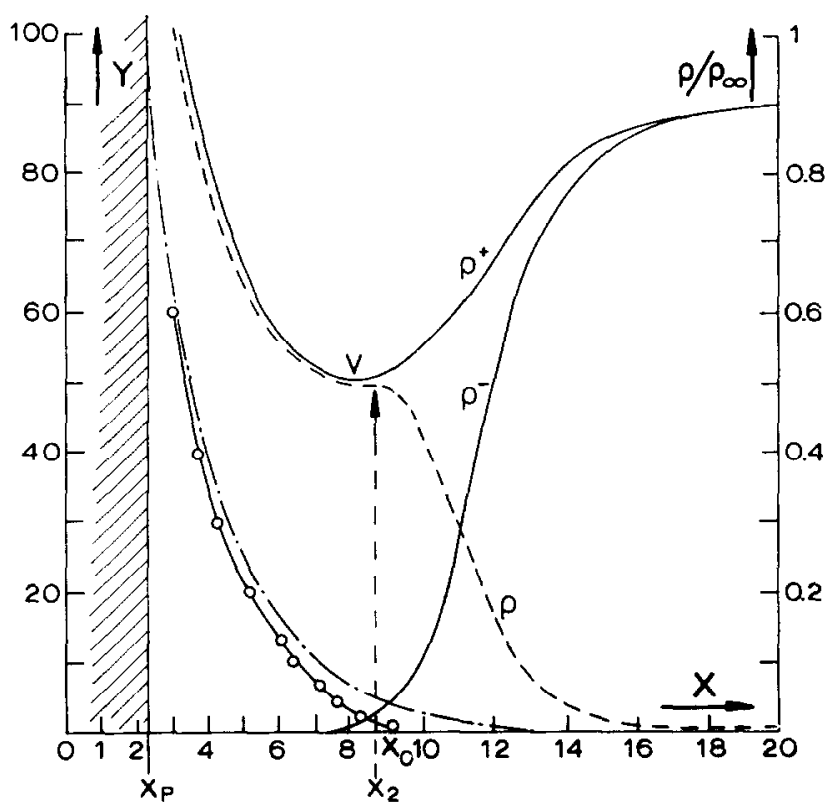

Fig. 3. Potential and space charge for a spherical probe with $p / 4 \pi=80$ calculated from the values given by Allen. ---- $=\rho^{+}-\rho^{-} ;-.-.=$potential as calculated by Allen; $O-O=$ a solution of the space-charge equation of Langmuir for $x_{p}=2.3$; $y_{p}=100$.

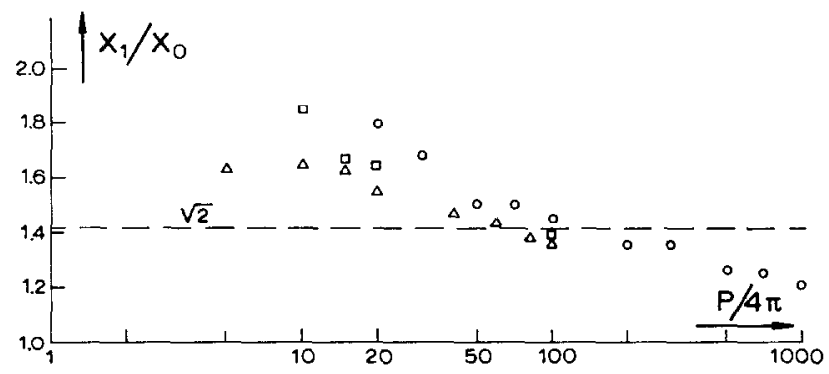

Fig. 4. The ratio of $x$, the radius of the plasma boundary at $y=0.5$ and $x_{0}$ the radius of the space charge region is given as a function of the current $p / 4 \pi$ to the probe. $x_{0}$ is calculated with help of the computer curves of Allen.

$$
\{y=100(0) ; y==60(\square) ; y=20(\Delta)\} \text {. }
$$

of $y$ and $x$ where the space charge is a maximum. We then calculate that for $x=x_{2}, y$ varies between 1.5 and 2.5 depending on $p$. Kagan takes the boundary at $x_{2}$ for $y_{2}=1.5$. We find then:

$$
x_{2}^{2}>p / 8 \pi \exp \left(-y_{2}\right) y_{2}^{1.5}>(0.41)^{-1} p / 8 \pi
$$


II. 4. Relation between current and voltage. From the equations (9) and (11) we calculate for the ratio $x_{1} / x_{2}$ :

$$
1<\left(x_{1} / x_{2}\right)^{2}<2.0
$$

with $y$ in the neighbourhood of 0.5 .

Kagan takes now $\left(x_{1} / x_{2}\right)^{2}=2$ and the replaces $x_{2}$ by $x_{0}$. Because this is a doubtful point we have calculated the ratio $\left(x_{1} / x_{0}\right)$ from the computer values of Allen e.a. for different $p$ and $y$. The results are given in fig. 4 . For $y=20$ and lower, and currents $p / 4 \pi=20 \rightarrow 700$, the difference between Kagan's approximation of 1.42 and the calculated ratios is within $10 \%$ which is quite good. The experiments, carried out in a helium discharge, are within the above given limits for current and voltage, so we shall use the value of $x_{1} / x_{0}=1.42$. Substituting this value in equation $(7 a)$, we find for the radius $x_{1}$ of the plasma boundary:

$$
x_{1}=1.41 x_{p}\left[1+\left\{7.44 p^{-1} y^{1.5}\right\}^{0.58} .\right.
$$

Elimination of $x_{1}$ from equation (13) and (9) yields the following relation between the probe current, probe voltage and ion density:

$$
y_{p}=(0.0134 p)^{0.67}\left\{(0.095 p)^{0.5} x_{p}^{-1}-1\right\}^{1.15}
$$

This formula gives good results for $y>5$, because otherwise the replacement of $x_{2}$ by $x_{0}$ in equation (13) is not justified. From formula (14) it is clear that, because $y_{p}$ cannot become negative,

$$
0.095 p>x_{p}^{2}
$$

which states that the probe current, $i^{+}-p I_{A}$, has to be bigger than the drift current across the boundary for which $x_{1}^{2}=2 x_{p}^{2}$.

II. 5. Comparison with other authors. We have compared formula (14) with the calculations of Allen e.a., Bernstein e.a. and Chen. The results are given in fig. 5 . The calculations of Allen and Bernstein agree with each other (there are some misprints in the article of Bernstein). Chen has calculated a more extensive range.

Our formula gives results which differ by only $5 \%$ from the computed curves, which is quite satisfactory in view of the fact that we have used a simplified picture. From equation (14) we calculate the ion density by substituting

$$
n_{0}=69^{2} T_{e}\left(x_{0} / x_{p}\right)^{2}
$$

which leads to the following equation:

$$
\begin{gathered}
n_{0}=4.51 T_{e} p / r_{p}^{2}\left\{1+3.21 y_{p}^{0.87} p^{-0.58}\right\}^{-2} \\
\left(n_{0} \text { in } \mathrm{m}^{-3}, T_{e} \text { in }{ }^{\circ} \mathrm{K}, r_{p} \text { in } \mathrm{m}\right) .
\end{gathered}
$$




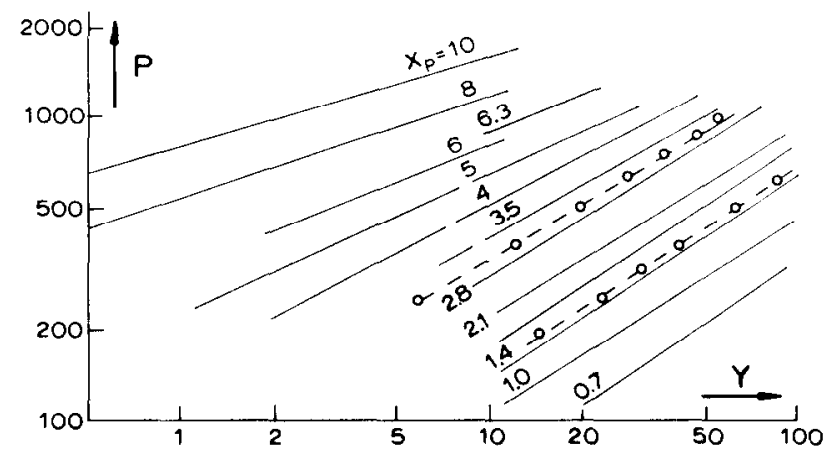

Fig. 5. Comparison of calculated values of the current as a function of potential. Left part of the figure calculations by Bernstein and Rabinowitz; right part Allen; $0-0-0$ our formula.

III. Measurements. The electron and ion densities are measured with three different spherical probes made from platinum in a low pressure helium discharge. The discharge is made in a glass tube with inner diameter of $50 \mathrm{~mm}$; oxide coated filaments are used as anode and cathode. The filaments are degassed thoroughly; the helium gas is purified by diffusing it through a heated quartz tube. The rest gas pressure of the system, after baking at $400^{\circ} \mathrm{C}$ is less than $10^{-8}$ torr. The measurements are performed at pressures of about 0.15 torr; tube currents of $0.5 \rightarrow 2 \mathrm{~A}$ are used. The three probes with diameters of 344 (I), 425 (II) and $585 \mu$ (III), can be moved radially in the tube.

The probe characteristic is measured with an $x-y$ recorder. A (slow) sweep voltage with a maximum value of $20 \mathrm{~V}$ in about $20 \mathrm{~s}$ is used to scan the characteristic around the plasma potential.

The temperature is calculated from the $\log i_{e}-V$ characteristic, in which the electron current $i_{e}$ is corrected for the ion contribution. This correction is performed by measuring the ion current over $200 \mathrm{~V}$, plotting these measurements on a log-log scale, which gives a straight line down to about $30 \mathrm{~V}$, and extrapolating this line to the desired voltages.

The electron density $n_{e}$ is calculated from the measured current at plasma potential and from the saturation current, following a method described by Freudenthal and Kinderdijk ${ }^{7}$ ).

The plasma potential, which is defined by the knee in the probe characteristic, is unambiguously defined by measuring the second derivative of the probe current. This is done by using two frequencies (500 and $666 \frac{2}{3} \mathrm{~Hz}$ ) together on the probe line, and measuring, with phase sensitive detection, the amplitude of the difference frequency $\left(166 \frac{2}{3} \mathrm{~Hz}\right)$ which is a measure for the second derivative (see Friar $\left.{ }^{8}\right)$ ). The plasma potential derived from the second derivative is compared with the potential derived from an iterative 
process also proposed by Freudenthal and Kinderdijk8). The two methods give the same plasma potential within $2 \%$.

The ion densities are calculated with help of formula (14), from the measured ion current at about 50 volts negative with respect to floating potential. We have checked the calculations by measurements at other voltages and the results differ only slightly; the biggest difference is $5 \%$. In calculating the ion density we have corrected the ion current for the secondary electron emission $\gamma_{i}$ caused by the helium ions on platinum. For $\mathrm{He}^{+}$on platinum there are no values known at the moment. Kaminsky ${ }^{9}$ ) suggest to take a value for $\mathrm{He}^{+}$on $\mathrm{W}$ or Mo which is about $28 \%$ for a clean surface. We have taken a value of $30 \%$ which means that the measured ion current is corrected with $22.5 \%$.

TABLE II

\begin{tabular}{|c|c|c|c|c|c|}
\hline $\begin{array}{c}\text { Probe } \\
\text { number }\end{array}$ & $\begin{array}{l}\text { pressure } \\
\text { in torr }\end{array}$ & $\begin{array}{c}\text { tube } \\
\text { current in } A\end{array}$ & $\begin{array}{l}\text { temperature } \\
\text { in }{ }^{\circ} \mathrm{K} \times 10^{3}\end{array}$ & $\begin{array}{c}n_{e} \times 1 \overline{0^{16}} \\
\mathrm{~m}^{-3}\end{array}$ & $\begin{array}{c}n_{i} \times 10^{16} \\
\mathrm{~m}^{-3}\end{array}$ \\
\hline I & 0.14 & 1.0 & 49 & 5.07 & $\overline{5.22}$ \\
\hline II & 0.14 & 1.0 & 63 & 5.95 & 5.20 \\
\hline III & 0.14 & 1.0 & 52 & 6.20 & 6.17 \\
\hline II & 0.14 & 0.5 & 74 & 2.62 & 2.60 \\
\hline II & 0.14 & 0.5 & 57 & 3.12 & 2.74 \\
\hline I & 0.21 & 0.85 & 42 & 3.39 & 4.04 \\
\hline III & 0.21 & 0.85 & 56 & 3.27 & 3.97 \\
\hline II & 0.22 & 1.0 & 64 & 6.14 & 6.90 \\
\hline I & 0.22 & 2.0 & 64 & 13.5 & 14.6 \\
\hline II & 0.10 & 1.0 & 73 & 3.12 & 2.92 \\
\hline I & 0.10 & 1.0 & 77 & 2.53 & 2.48 \\
\hline II & 0.10 & 1.5 & 71 & 4.98 & 4.38 \\
\hline I & 0.10 & 1.5 & 80 & 4.03 & 3.85 \\
\hline III & 0.10 & 0.7 & 59 & 2.32 & 2.14 \\
\hline II & 0.10 & 2.0 & 54 & 13.2 & 15.2 \\
\hline
\end{tabular}

The calculated electron and ion densities are given in table II. The measurements were performed under different conditions of pressure and tube current. As can be seen from the results the biggest difference is $20 \%$; the standard deviation is $10 \%$.

IV. Conclusion. A new analytical formula is derived, equation (16), with which it is possible to calculate directly the ion density from a measurement of the ion current and voltage, if the electron temperature is known. The ion current must be corrected for the secondary emission of the probe surface. The ion densities so calculated are in good agreement with measurements of the electron densities (measured in the positive column of a helium discharge), which were calculated from the current at plasma potential and from the saturated electron current. The results indicate that 
the plasma density may be calculated with a good accuracy also from the ion saturation current.

Acknowledgement. The authors want to thank Professor Dr. J. A. Smit for many helpful discussions. They also wish to express their gratitude to Dr. T. Matitti and Ir. A. Vermeer for their comments on this paper.

This work is part of the research program of the association agreement of Euratom and the "Stichting voor Fundamenteel Onderzoek der Materie" (F.O.M.) with financial support from the "Nederlandse Organisatie voor Zuiver Wetenschappelijk Onderzoek" (Z.W.O.) and Euratom.

Received 1-8-66

\section{REFERENCES}

1) Allen, J. E., Proc. Phys. Soc, B70 (1957) 297 and Proc. Phys. Soc. $\mathbf{8 3}$ (1964) 177.

2) Bernstein, I. B. and Rabinowitz, I. N., Phys. Fluids 2 (1959) 112.

3) Chen, F. F., J. Nucl. Energy C (Plasma Physics) 7 (1965) 47.

4) Kagan, Yu. M. and Perel, V. I., Soviet Physics Uspekhi 767 (1964).

5) Langmuir, I., Collected works III, IV and V, London, Pergamon Press (1961).

6) Ollendorff, F., Elektronik freier Raumladungen, Wien, Springer Verlag (1957).

7) Freudenthal, J. and Kinderdijk, H. M. J., Physica 32 (1966) 158.

8) Friar, E. M., Report of the Wright Patterson A. F. Base Ohio 1964.

9) Kaminsky, M., Atomic and ionic impact phenomena on metal surfaces, Berlin, Springer Verlag (1965), p. 296. 